\title{
Polarized light emission from grain boundaries in photovoltaic silicon
}

\author{
T. W. Lin, L. P. Rowe, A. J. Kaczkowski, G. P. Horn, and H. T. Johnson \\ Department of Mechanical Science and Engineering \\ University of Illinois at Urbana-Champaign \\ email: htj@,illinois.edu
}

\begin{abstract}
Some crystalline defects in photovoltaic silicon have deleterious effects on the energy conversion efficiency of the material. Distinguishing the harmful defects from the benign defects is a critical problem in the mechanics of materials for solar energy conversion. Interestingly, the visible light absorbed by silicon in the same part of the solar spectrum that is used to generate photocurrent, can also excite photoluminescence, which may be used to generate images of the microstructure. Slightly longer wavelengths in the near infrared (IR) may be used to measure strain in the material via photoelastic (PE) imaging. These two imaging modalities have recently been combined in a single instrument, and we show here the additional capability to identify and categorize defects directly by capturing the narrow band of photoluminescence emitted by regions of high dislocation density. We use this method to show that dislocations arranged in low angle grain boundaries emit polarized light, while dislocation structures in neighboring high angle grain boundaries do not emit polarized light. This capability may form the basis for nextgeneration, full-field optomechanics-based characterization of materials for solar energy conversion.
\end{abstract}

\section{Introduction}

Polycrystalline silicon, or multi-Si, is the active material in the vast majority of photovoltaic solar cells, because it is inexpensive to produce, and its band gap is closely matched to the energy of light in the solar spectrum. Photovoltaic grade silicon contains many more defects than microelectronic grade silicon, including grain boundaries, inclusions, and dislocations, some of which have deleterious effects on the optical 


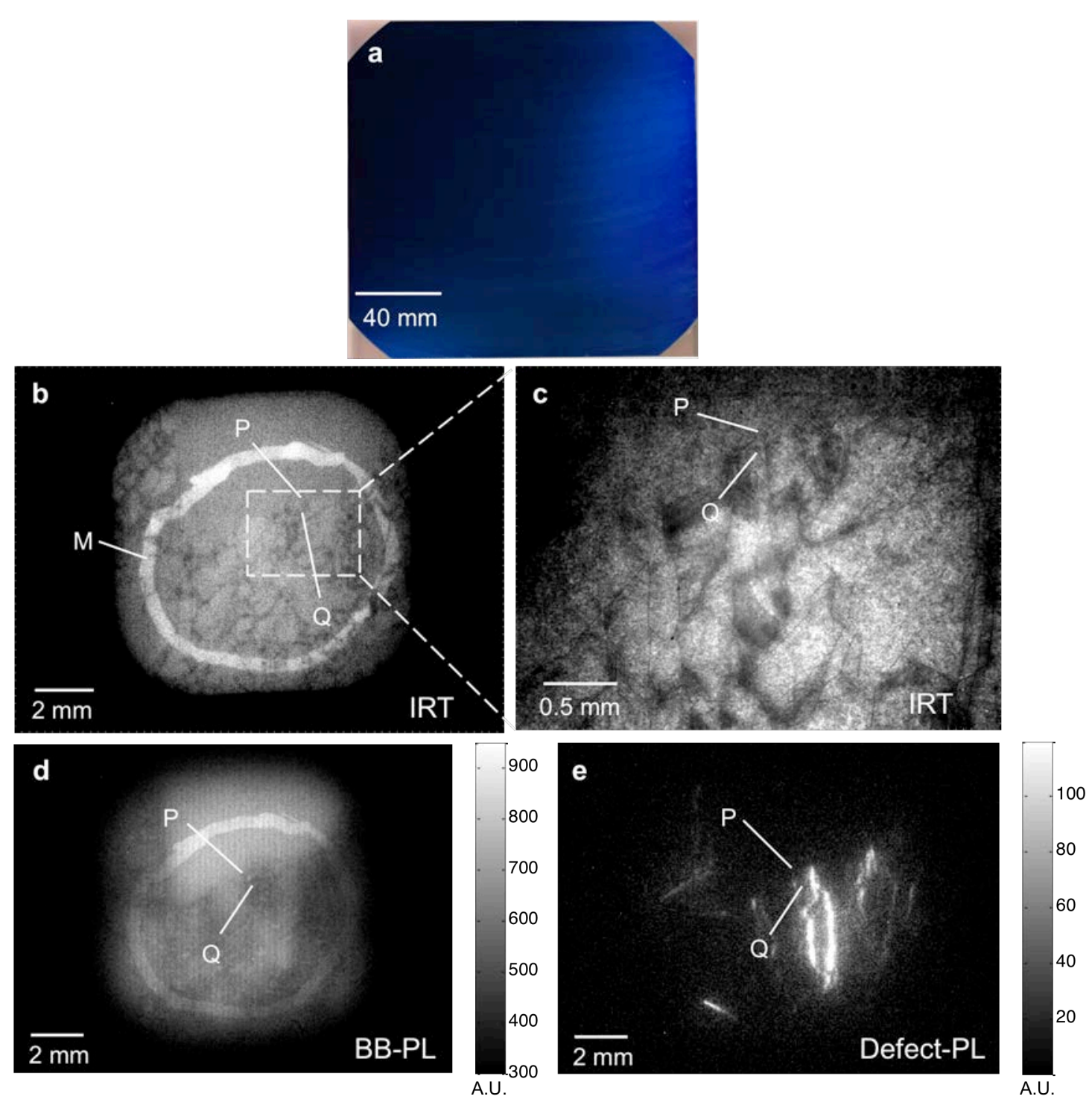

Figure 1. (a) Photograph of a multi-Si PV wafer with nitride anti-reflection coating; (b) IRT image of a selected area in the wafer; ' $M$ ' is a ring made on the wafer surface for image location, location ' $\mathrm{P}$ ' is near the edge of a clean grain, and location ' $\mathrm{Q}$ ' is within an area with defect structures. (c) Microscopic IRT image of the area marked by dashed box in (b). (d) Near-IR PL image of the same section of the wafer. The emission is dominated by band edge (or "band-to-band") recombination. Areas of higher defect density have lower band edge PL intensity. (e) Defect-band PL emission, at a slightly longer wavelength, obtained by applying a long pass filter to the signal from (c). The PL images and the imaging method are presented in greater detail in [16].

absorption efficiency of the material [1]. The PV industry uses infrared transmission 
(IRT) imaging as a simple quality control method to eliminate sub-standard material before as-cut wafers enter the next PV processing step. IRT imaging reveals the features in a PV wafer by the intensity contrast due to differing amounts of transmitted and scattered light [2,3]. Figure 1a shows a typical $156 \mathrm{~mm}$ x $156 \mathrm{~mm}, 200 \mu \mathrm{m}$ thick multi-Si PV wafer after the anti-reflection coating (ARC) has been deposited on the front surface. High magnification infrared images in Figure $1 \mathrm{~b}$ through e are captured with the back surface (without ARC) facing the camera. Figure 1b shows an IRT image of a $10 \mathrm{~mm}$ square portion of this multi-Si PV wafer using broad band IR illumination with peak wavelength around $1150 \mathrm{~nm}$. In Figure 1b, a ring made on the surface of the wafer using a marker is labeled as ' $M$ ' and used to locate the specific region of interest. The upper part of the image shows a grain with a relatively uniform IRT intensity (location 'P') that can be distinguished from the neighboring area with darker, irregular fine features (location 'Q'). Similar dark features, which may be defects including impurities, precipitates, or crystalline defects such as dislocation clusters, also exist in other parts of the wafer. Figure $1 \mathrm{~b}$ is obtained using a $5 \mathrm{X}$ microscopic lens to more closely examine the region in the dashed box, which includes the grain with uniform IRT intensity (location 'P') and the area with defects (location ' $Q$ ').

The relative quantity of these defect structures, as identified using IRT, is considered to be a rough indicator of wafer quality. Wafers typically only contain single grains through their thickness. Grain size is regarded as an important factor, since smaller grain size introduces more grain boundaries, which are potentially deleterious features $[4,5]$. However, the presence of defect structures or grain boundaries alone does not directly correlate to reduced solar cell performance. To gain a better understanding of the wafer quality, it is essential to understand the link between the defects and the solar cell optoelectronic properties such as carrier lifetime [6,7].

Band-to-band photoluminescence (PL) imaging has been widely adopted as a means to infer solar cell carrier lifetime [8-11]. Band-to-band PL, which is emitted when the electron-hole recombination process releases a photon with an energy equal to the 1.1 $\mathrm{eV}$ band gap energy, has been successfully correlated to the carrier lifetime [6]. The presence of crystalline defects can significantly reduce the band-to-band PL intensity because these defects introduce energy levels that allow the electron-hole pair to 
recombine with PL emission at about $0.8 \mathrm{eV}$ at room temperature $[12,13]$. The defectrelated emission is referred to as defect-band PL. The PL image in Figure $1 \mathrm{~d}$ is from the same field of view as in Figure $1 \mathrm{~b}$ but the signal is filtered to pass only the band-to-band emission. The band-to-band PL intensity is uniform and higher in location 'P'. The area with more defect structures in location 'Q' shows lower band-to-band PL intensity. The PL image shown in Figure 1e is created by allowing only the defect-band PL to pass. The emission from defect structures can be clearly identified in location 'Q', while none is observed in location ' $\mathrm{P}$ '.

While band-to-band PL is not associated with a particular optical polarization state, defect-band PL is highly polarized. Ostapenko et al., using a scanning method for measuring PL as opposed to a full-field method, conclude that the polarization direction is perpendicular to nearby grain boundaries, and suggest that the stress state near the grain boundaries is causing the anisotropic polarization of defect-band PL from the dislocations [12]. Peloso et al. use a related technique, electroluminescence (EL) imaging, to study the defects in a PV wafer and observe increased polarized luminescence in areas with higher dislocation densities [14]. They make this measurement with full-field EL detection, but without measuring local stresses; their suggestion, contradictory to that of Ostapenko et al., is that polarization of luminescence from the defects is due to a quantum mechanical effect at the dislocation core, which introduces a restriction on the polarization vector of the emitted light. These studies do not distinguish the types of defects in their measurements, or the relationship between the dislocation type, the dislocation microstructure, and the grain boundary structure. Kato et al., using a small field-of-view PL measurement system (that does not include simultaneous stress measurement), show a 90-degree difference in defect-band PL polarization angle between tilt and twist grain boundaries [15]. This recent work suggests that dislocation ordering in grain boundary structures may be responsible for polarization of the defect-band luminescence, but it does not consider the difference between small angle and high angle grain boundaries.

Multi-Si PV wafers are processed according to a standard procedure that leads to unique microstructural and defect content. After the wafers are first cut from an ingot via wire-sawing, the surface is etched to remove saw damage. At this stage, the wafer 
surface becomes rough and etch pits may appear if there are dislocation lines intersecting the surface. Figure 2a shows a scanning electron microscopy (SEM) image of a location near the area in Figure 1b, with marks ' $\mathrm{P}$ ' and ' $\mathrm{Q}$ ' indicating the same locations. Location ' $\mathrm{P}$ ' is characterized by a uniform intensity, while location ' $\mathrm{Q}$ ' has many brighter, finer, and more irregular features. These are the same features that appear dark in Figure $1 b$. Figure $2 \mathrm{~b}$ shows the SEM image at a $150 \mathrm{X}$ magnification in the area marked by dashed lines in Figure $2 \mathrm{a}$ and reveals the dislocation etch pits that form pileups. At this magnification, the rough surface texture can be observed. A linear feature in the middle along the horizontal direction is observed, separating locations ' $\mathrm{P}$ ' and ' $\mathrm{Q}$ '. An 850X magnification SEM image in Figure 2c focuses at the horizontal boundary, which shows no etch pit pile-ups along the boundary. However, in the defective area (location 'Q'), two linear inclined features are observed with dislocation etch pits lined up along the features. These SEM images confirm that the dark irregular features observed using the IRT imaging are defect structures formed by dislocations. Dislocation
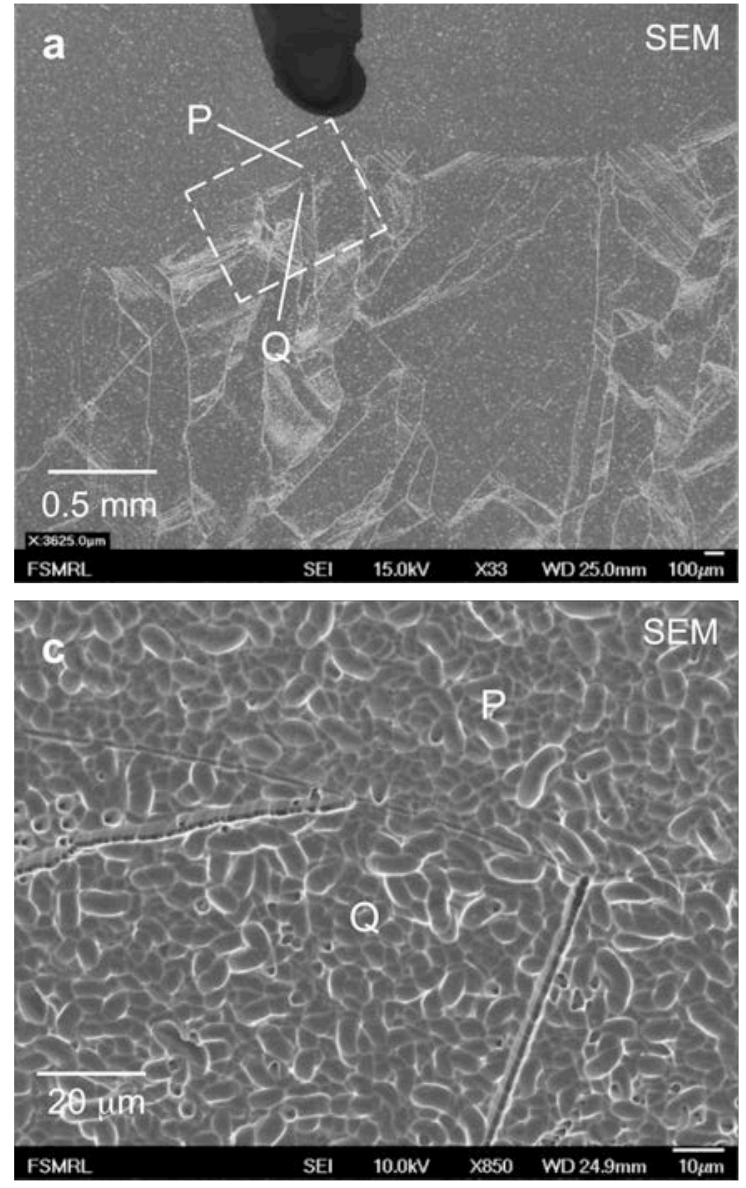

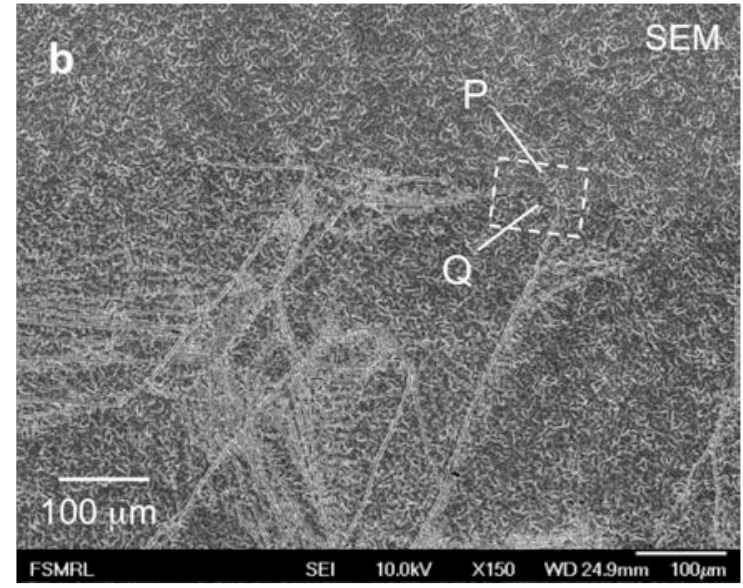

Figure 2. SEM images of the same multi-Si PV wafer in Figure 1 show (a) a field of view that includes location ' $\mathrm{P}$ ' in a clean grain and location ' $Q$ ' in a defective area; (b) $150 \mathrm{X}$ image in the area marked by dashed lines, showing that the defect structures consist of dislocation etch pits; (c) $850 \mathrm{X}$ image shows etch pits arranged along linear features near location ' $Q$ ', but none along the boundary separating 'P' and 'Q'. 
structures of this kind have been identified as potential sources of reduced solar cell performance [1].

In the present work, we investigate defect structures in a multi-Si PV wafer using a novel multi-modal infrared imaging system, which can perform various imaging analyses, including IRT imaging, infrared photoelastic (PE) imaging, band-to-band PL, and polarized defect-band PL imaging. We compare the PL signals from grain boundaries with varying degrees of misorientation, including both low angle $(<10$ degrees) and high angle grain boundaries. By comparing information provided by each of these analyses along with high magnification electron microscopy, the optical properties of the crystal defects and their impact on PV wafer quality can be better understood.

\section{Methods}

We have recently developed a multi-modal infrared inspection system for imaging silicon PV wafers [16]. The system uses a broadband InGaAs IR camera, which, in addition to generating standard IR transmission images, can be used for IR photoelastic inspection, and both band-to-band and defect-band PL imaging with polarization resolution. When operated in PE mode, this system is based on a commercially available PE instrument known as an infrared grey-field polariscope (IR-GFP) built by StressPhotonics Inc., using a circularly polarized light source with a peak at $1150 \mathrm{~nm}$. The IR-GFP measurement determines the linear retardation induced by mechanical strain, between the two mutually orthogonal in-plane principal strain directions [17]. Illumination for the PL imaging is provided by a light-emitting diode (LED) source with a peak wavelength of $630 \mathrm{~nm}$. 


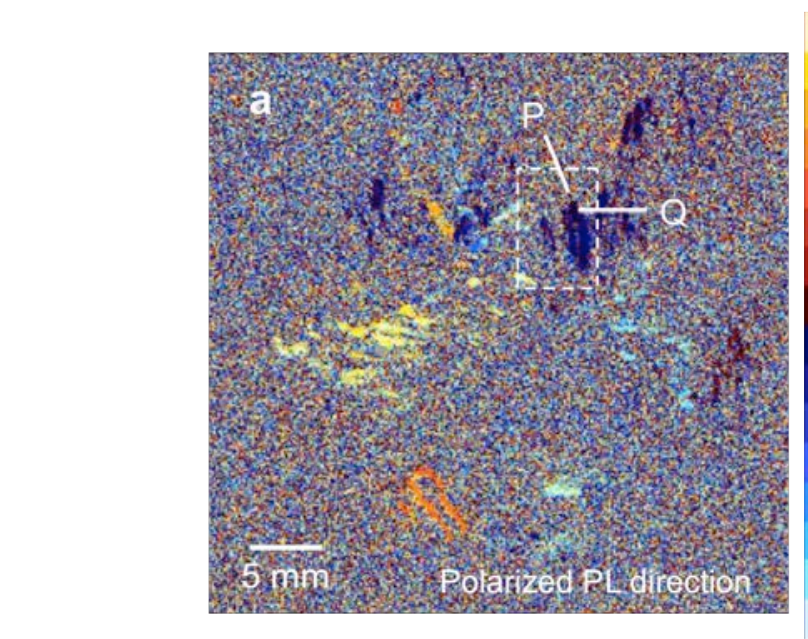

Figure 3. (a) Polarized defectband PL image for a large section of the multi-Si wafer. The dashed box shows the region containing locations ' $\mathrm{P}$ ' and ' $\mathrm{Q}$ '. (b) Microscopic IRT image shows the detail from the box in (a), where ' $P$ ' is in a defect-free grain, and ' $Q$ ' in an area with many defect structures. (c) Microscopic IR-GFP 66.0 measurement shows the strain78.0 induced retardation near location .90 .0 P and Q.
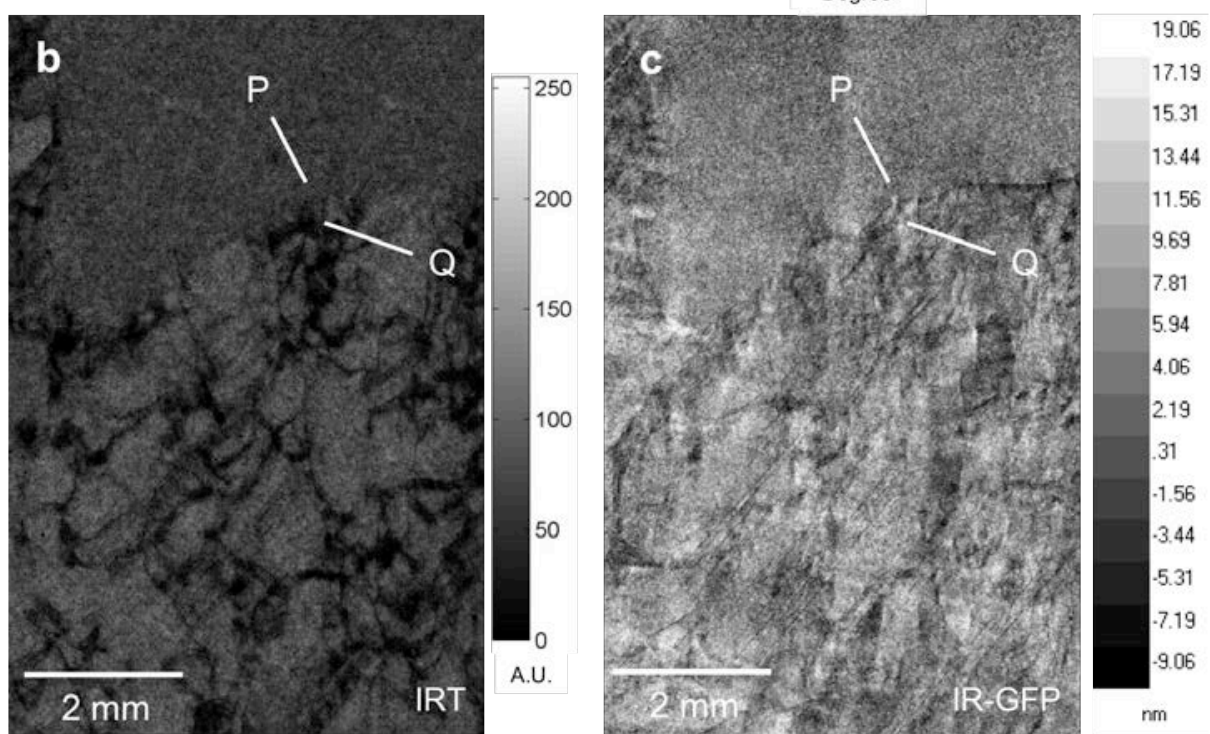

The band-to-band PL image in Figure 1c is obtained with a $1100 \mathrm{~nm}$ long pass filter in front of the camera with a $29 \mathrm{~ms}$ image acquisition time and a resolution of 0.025 $\mathrm{mm}$ per pixel. The band-to-band PL image captures the carrier recombination activity that emits photon energy near the silicon band gap at $1.1 \mathrm{eV}$, or $1150 \mathrm{~nm}$ in wavelength. Comparing the IRT image in Figure $1 \mathrm{~b}$ and the band-to-band PL image Figure 1d, it is possible to identify grains with more uniform IRT intensity and fewer defect structures, which tend to have a stronger band-to-band PL emission, such as at location 'P'. In contrast, location ' $Q$ ' has many defect structures in the grain and shows a relatively lower band-to-band PL intensity.

Defect-band PL is associated with an emission peak around $1500 \mathrm{~nm}$ at room temperature. The LED light source in the PL imaging system excites both the band-toband and the defect-band PL signal. Therefore, a long pass optical filter is needed to 
block the PL wavelength shorter than $1300 \mathrm{~nm}$, leaving only the PL peak around 1500 $\mathrm{nm}$. This image is referred to as the defect-band PL image. The defect-band PL in Figure 1e is obtained using the InGaAs infrared camera with an acquisition time of $29 \mathrm{~ms}$ and a resolution of $0.025 \mathrm{~mm}$ per pixel. A dark image with the LED turned off is captured as the reference image and is subtracted from the measured PL image to reduce electronic noise in the sensor. The regions emitting lower band-to-band PL in Figure 1d show higher defect-band PL in Figure 1e. For example, location ' $\mathrm{P}$ ' shows a high bandto-band PL intensity at 650 units, but low defect-band intensity. Location 'Q', in contrast, has defect-band PL intensity as high as 100 units, and band-to-band PL intensity at only 330 units. While the occurrence of defect features in Figure $1 \mathrm{~b}$ matches the location lacking of band-to-band PL in Figure 1d, the intensities between the band-toband and the defect-band PL are not inversely correlated. Therefore, the band-to-band and defect-band emission provide different aspects of defect properties and should be analyzed together. The defect-band PL image captures a defect type that is not perceivable from either the IRT or the band-to-band PL images. These strong defectband signals are optically polarized and often appear as short segments in the defect-band PL image.

To determine the polarization state of the PL emitted from defect structures, a video processing algorithm is used along with a rotating polarizer in front of the InGaAs camera [16]. During this measurement, the camera collects up to 160 images as the analyzer continuously rotates through 16 different positions. By comparing the PL intensity in the defect-band wavelength range for each pixel to the analyzer angle, the method makes it possible to achieve significant noise reduction. The polarization angle as a function of position is shown in Figure 3a. This data set and the details of the experimental method are presented in [16]. The regions of high intensity defect-band PL, including those labeled as ' $\mathrm{P}$ ' and ' $\mathrm{Q}$ ' in Figure 3a, appear as short line segments. The polarization angle of the emitted light is clustered around several characteristic angles ranging from -90 degrees to +90 degrees.

The approach presented here provides noise reduction through signal processing techniques including subtraction of the dark image as well as the averaged ambient light from multiple polarized PL images. Thus, the signal to noise ratio for the polarized part 
of the PL emission is enhanced, while non-polarized noise and emission are rejected. The ability of this imaging tool to resolve the polarized magnitude and the polarization direction is limited by the noise signal from stray light and the thermal noise in electronics. The minimal resolvable signal intensity depends on the background intensity where there is no defect PL emission present. When 10 analyzer revolutions are required during acquisition (total 160 images), the background standard deviation is 15 units, which is small compared to the maximum and minimum intensities from defect PL emission of about \pm 300 units. Compared to the conventional approach, where the analyzer has to be manually set to a desired angle [15], the imaging tool presented here takes advantage of the digitized controller and can complete a full-wafer measurement in less than a minute.

\section{Results}

To further understand the relation between the defect structures and the PL emission, the region of materials shown in Figure 3a, containing the locations ' $\mathrm{P}$ ' and ' $Q$ ', is investigated using both IRT and IR-GFP imaging. Figure $3 \mathrm{~b}$ shows a microscopic IRT image created from 8 images stitched together to create a larger field of view. This procedure is also done for the IR-GFP image in Figure 3c, showing the same field of view. The upper part within the box in Figure 3a shows no defect-band PL signal near location 'P', which corresponds to the defect-free grain in the IRT image. There is a strong defect-band PL emission near location ' $Q$ ' in Figure 3a, which corresponds to the dark, irregular defects in the IRT image. There are strain signatures associated with these defects, as shown in Figure 3c. The strain-induced retardation varies significantly around location ' $\mathrm{Q}$ ' relative to location 'P', where the retardation remains relatively uniform in the defect-free grain. The non-uniform strain field is a signature associated with crystal defects found in silicon wafers [18]. 

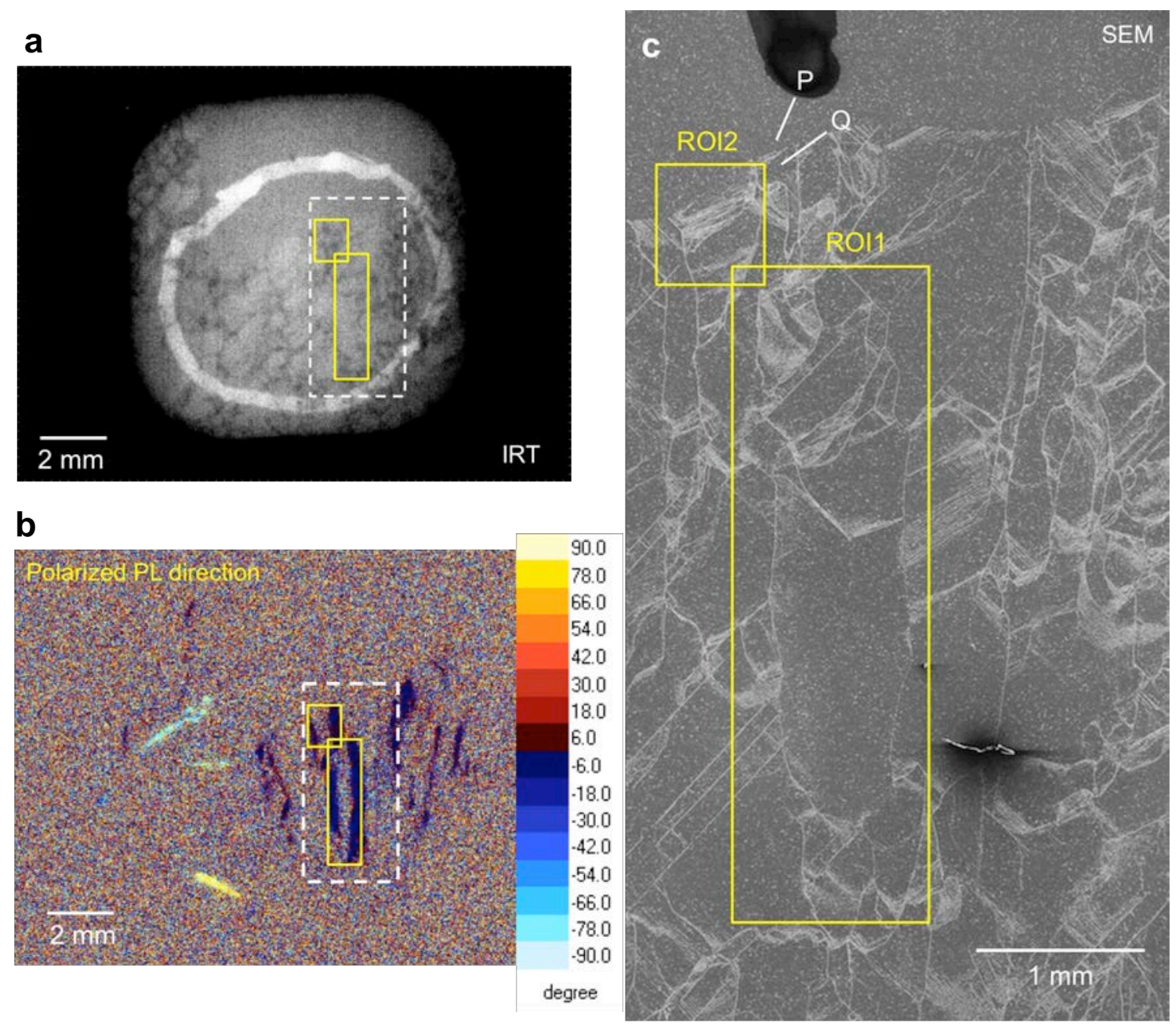

Figure 4. (a) Same IRT image from Figure 1a; (b) direction of polarized defect-band PL; (c) SEM image showing the detail in white dashed box marked in a and $b$.

The polarized PL imaging method reveals the optical properties of defect structures in a wafer, which makes it possible to distinguish different defect structures seen in the IRT images in Figure 4a. Figure 4b shows the direction image of the defectband PL emission for the same field of view. The polarized defect-band PL features near location ' $Q$ ' from Figure 3a are presented with details in Figure 4b, where parallel segments are clearly resolved and shown to have zero-degree polarization angle. To investigate the origin of the polarized defect-band PL, an SEM image shown in Figure 4c is obtained for detailed microstructures within the white dashed box marked in Figure 4a and $\mathrm{b}$. The yellow solid boxes marked in Figure $4 \mathrm{a}, \mathrm{b}$, and $\mathrm{c}$ indicate the regions of interest for electron backscatter diffraction (EBSD) measurements, which detect the electron diffraction pattern scattered off the surface to determine the local crystal orientation. 
As shown in Figure 4c, ROI1 is long and rectangular, and includes dislocation clusters surrounding a relatively defect-free area in the middle. In Figure 4b, ROI1 contains two long, vertical regions with polarized PL signal. In Figure 4c, the upper part of ROI2 includes a uniform grain with no dislocation clusters (location 'P'). The lower part of ROI2 includes an area with an increased concentration of defects (location 'Q'). ROI2 also includes two short, vertical regions with polarized PL signal, as shown in Figure $4 b$.

The EBSD results for ROI1 are shown in Figure 5. The inverse pole figures (IPF) indicate the crystal orientation along the sample coordinates, where $\mathrm{Z}$ is the surface normal, $\mathrm{Y}$ is the vertical direction, and $\mathrm{X}$ is the horizontal direction. The two vertically oriented grain boundaries (GBs) can be observed from the EBSD results in Figure 5a, which suggest that they are low angle GBs. A low angle GB refers to a boundary between two grains with their crystal lattice misorientation angle typically smaller than 10 degrees, which can be commonly found in a multi-Si PV wafer [19]. The misorientation angles for the small angle GBs on the left and on the right are approximately 3.1 and 7.1 degrees, respectively. The crystal lattice direction [535] along the $\mathrm{Z}$-direction for the grain in the middle is the same as its neighboring grains. This suggests that the crystal lattice is misoriented by rotation of the middle grain about the Zaxis, creating low angle tilt GBs around the grain. 
In Figure 5, the EBSD results for ROI2 show that there are three GBs. The two long vertical GBs shown in Figure 5 are both low angle GBs with misorientation angles of 3.1 degrees on the left and 4.9 degrees on the right, respectively. These GBs physically correspond to the locations emitting polarized PL signals in Figure 4b.
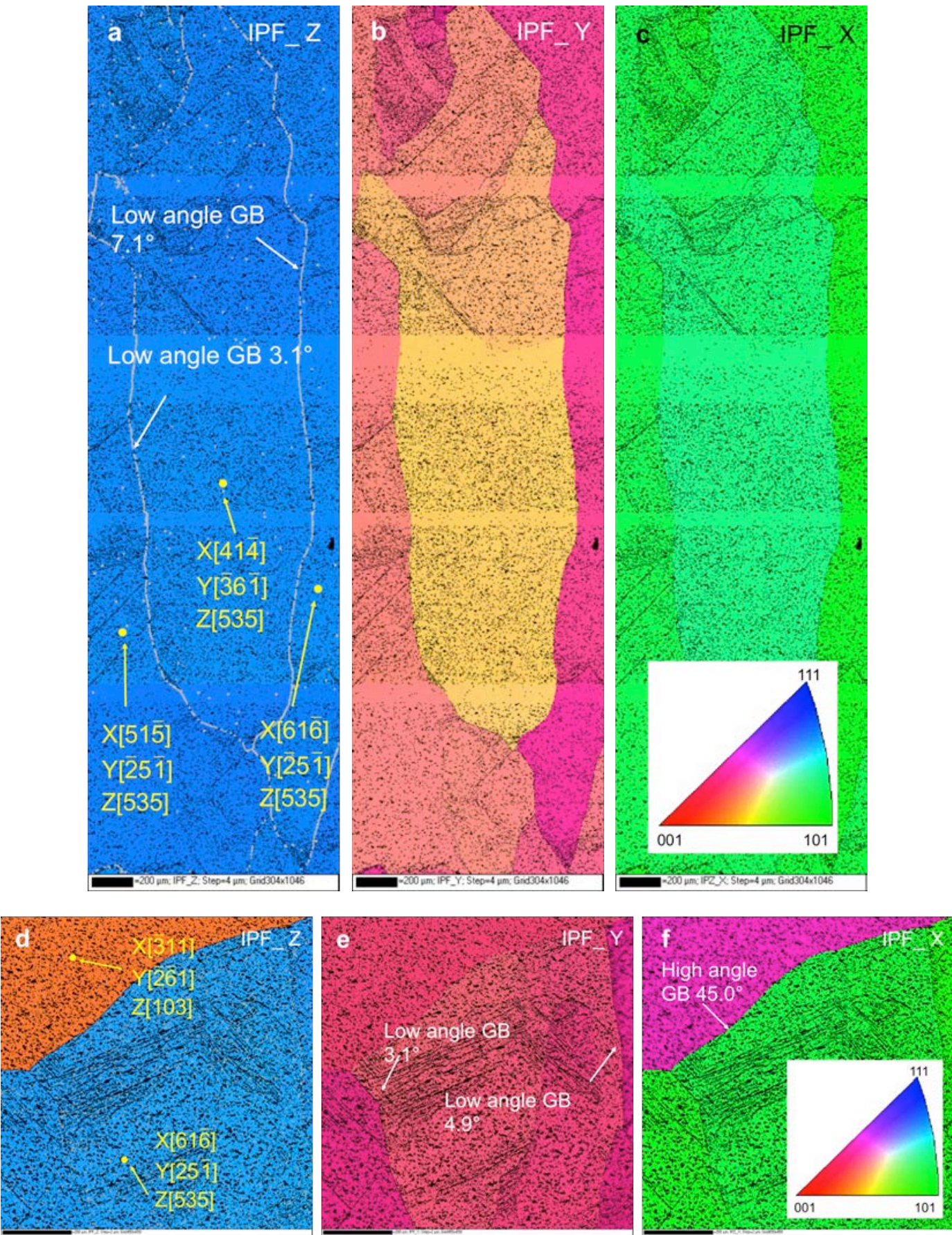

Figure 5. (top: a,b,c) EBSD measurement in ROI1 indicating the crystal orientation along the sample coordinate (a) Z, or the surface normal direction; (b) Y, or the vertical direction; (c) X, or the horizontal direction. (bottom: d,e,f) EBSD measurement in ROI2 indicating the crystal orientation along the sample coordinate (d) Z, or the surface normal direction; (e) Y, or the vertical direction; (f) $\mathrm{X}$, or the horizontal direction. 
However, the crystal orientations in the upper part and the lower part of Figure 5 are not the same. The GB separating the two grains with large mismatch is a high angle GB, which refers to the GB associated with a misorientation angle larger than 20 degrees; here the misorientation is 45.0 degrees. By comparing this location to the equivalent location in Figure $4 b$, it is apparent that the high angle GB does not emit a polarized PL signal.

These careful observations of crystal orientation and grain structure suggest that polarized PL emission is associated with low angle GBs in the wafer. The polarization effect is detected only at the grain boundaries, but not at all grain boundaries. In particular, when the dislocation structures form a low angle tilt GB, the polarized PL signal is spatially correlated to these GBs, suggesting that the stress state alone may not be responsible for the polarization effect, as proposed by Ostapenko et al. [12]. This polarization effect is not observed at all grain boundaries, thus the geometrical effect identified by Peloso et al [14] is also not solely responsible for the effect. Our result is consistent with the recent observations of Kato et al. [15] who report on the polarized nature of defect-band PL emission for both tilt and twist low angle GBs. However, our results further expand the understanding of these effects by showing that nearby high angle grain boundaries are not associated with any detectable polarized PL.

We also find defect structures with similar polarized defect-band PL emission in a batch of 10 other multi-Si PV wafers. We find no strong defect-band emission at the dislocation structures that do not form a boundary. For example, a dense dislocation cluster can be seen in the middle of ROI2 in Figure 4a, surrounded by the high angle GB and the two low angle GBs, but in Figure 4b, this dislocation cluster does not emit a detectable polarized PL signal. This finding is significant because of the current interest in understanding the role of dislocation clusters in PV silicon,[20] which are correlated with reduced carrier lifetime and thus reduced energy conversion efficiency in silicon solar cells.

\section{Summary and Conclusions}

High quality material generates strong band edge photoluminescence, while defective material, containing high dislocation densities, generates strong defect-band photoluminescence. We have confirmed that low angle grain boundaries generate 
polarized defect-band photoluminescence, while other grain boundaries do not. Low angle grain boundaries are found in regions with lower band-to-band PL emission, indicating their impact on the carrier lifetime. The ability to distinguish the optoelectronic signatures of various grain boundaries and dislocation structures could provide a significant advance in rapid nondestructive characterization of PV materials, where some defects result in deleterious effects on energy conversion efficiency, while others do not.

\section{Acknowledgement}

The authors acknowledge the support of National Science Foundation grant CMMI-13-00466.

\section{References}

[1] Sopori B, Mehta V, Devayajanam S, Seacrist M, Shi G, Chen J, et al. Defect Generation and Propagation in Mc-Si Ingots: Influence on the Performance of Solar Cells. Solid State Phenom (2013) v205-206, 55-64.

[2] Demant M, Welschehold T, Oswald M, Bartsch S, Brox T, Schoenfelder S, et al. Microcracks in Silicon Wafers I: Inline Detection and Implications of Crack Morphology on Wafer Strength. IEEE J Photovoltaics (2016) v6, 126-35.

[3] Stokkan G, Hu Y, Mjøs O, Juel M. Study of Evolution of Dislocation Clusters in High Performance Multicrystalline Silicon. Sol Energ Mat Sol C (2014), 1-7.

[4] Yang C. Effect of Sub-Grains and Crystal Defects on Monolike Si Solar Cell Performance. Mater Sci Appl (2013) v04, 103-8.

[5] Ryningen B, Stokkan G, Kivambe M, Ervik T, Lohne O. Growth of Dislocation Clusters during Directional Solidification of Multicrystalline Silicon Ingots. Acta Mater (2011) v59, 7703-10.

[6] Giesecke JA, Schubert MC, Michl B, Schindler F, Warta W. Minority Carrier Lifetime Imaging of Silicon Wafers Calibrated by Quasi-Steady-State Photoluminescence. Sol Energ Mat Sol C (2011) v95, 1011-8.

[7] Sinton RA, Cuevas A. Contactless Determination of Current-voltage Characteristics and Minority-Carrier Lifetimes in Semiconductors from QuasiSteady-State Photoconductance Data. Appl Phys Lett (1996) v69, 2510.

[8] Trupke T, Bardos RA, Schubert MC, Warta W. Photoluminescence Imaging of Silicon Wafers. Appl Phys Lett (2006) v89, 044107.

[9] Johnston S, Guthrey H, Yan F, Zaunbrecher K, Al-Jassim M, Rakotoniaina P, et al. Correlating Multicrystalline Silicon Defect Types Using Photoluminescence, Defect-Band Emission, and Lock-in Thermography Imaging Techniques. IEEE $J$ Photovoltaics (2014) v4, 348-54.

[10] Haunschild J, Glatthaar M, Demant M, Nievendick J, Motzko M, Rein S, et al. Quality Control of as-Cut Multicrystalline Silicon Wafers Using Photoluminescence Imaging for Solar Cell Production. Sol Energ Mat Sol C (2010) v94, 2007-12. 
[11] Kiliani D, Micard G, Steuer B, Raabe B, Herguth A, Hahn G. Minority Charge Carrier Lifetime Mapping of Crystalline Silicon Wafers by Time-Resolved Photoluminescence Imaging. $J$ Appl Phys (2011) v110, 054508.

[12] Ostapenko S, Tarasov I, Kalejs JP, Haessler C, Reisner E-U. Defect Monitoring Using Scanning Photoluminescence Spectroscopy in Multicrystalline Silicon Wafers. Semicond Sci Technol (2000) v15, 840-8.

[13] Ostapenko S, Romero M. Defect Mapping in Full-Size Multi-Crystalline Si Wafers. Eur Phys J Appl Phys (2004) v27, 55-8.

[14] Peloso MP, Hoex B, Aberle AG. Polarization Analysis of Luminescence for the Characterization of Silicon Wafer Solar Cells. Appl Phys Lett (2011) v98, 171914.

[15] Kato G, Tajima M, Toyota H, Ogura A. Polarized Photoluminescence Imaging Analysis around Small-Angle Grain Boundaries in Multicrystalline Silicon Wafers for Solar Cells. Jpn J Appl Phys (2014) v53, 080303.

[16] Lin T, Rowe LP, Kaczkowski AJ, Horn GP, Johnson HT. Polarization-Resolved Imaging for Both Photoelastic and Photoluminescence Characterization of Photovoltaic Silicon Wafers. submitted to Exp Mech (2016).

[17] Horn G, Lesniak J, Mackin T, Boyce B. Infrared Grey-Field Polariscope: A Tool for Rapid Stress Analysis in Microelectronic Materials and Devices. Rev Sci Instrum (2005) v76, 045108.

[18] Lin T-W, Horn GP, Johnson HT. Quantitative Infrared Photoelasticity of Silicon Photovoltaic Wafers Using a Discrete Dislocation Model. J Appl Mech (2015) v82, 011001.

[19] Funakoshi M, Ikeno N, Tachibana T, Ohshita Y, Arafune K, Ogura A. RoomTemperature Photoluminescence Evaluation of Small-Angle Grain Boundaries in Multicrystalline Silicon. Jpn J Appl Phys (2014) v53, 112401.

[20] Ryningen B, Stokkan G, Kivambe M, Ervik T, Lohne O. Growth of Dislocation Clusters during Directional Solidification of Multicrystalline Silicon Ingot. Acta Mat (2011) v59, 7703-7710. 